\title{
Collective motion of bacteria in two dimensions
}

\author{
Yilin $\mathbf{W u}^{*}$ \\ Shenzhen Research Institute, The Chinese University of Hong Kong, Shen Zhen 518027, China; Department of Physics, The \\ Chinese University of Hong Kong, Hong Kong SAR 999077, China \\ * Correspondence: ylwu@phy.cuhk.edu.hk
}

Received September 23, 2015; Revised November 23, 2015; Accepted November 27, 2015

\begin{abstract}
Collective motion can be observed in biological systems over a wide range of length scales, from large animals to bacteria. Collective motion is thought to confer an advantage for defense and adaptation. A central question in the study of biological collective motion is how the traits of individuals give rise to the emergent behavior at population level. This question is relevant to the dynamics of general self-propelled particle systems, biological self-organization, and active fluids. Bacteria provide a tractable system to address this question, because bacteria are simple and their behavior is relatively easy to control. In this mini review we will focus on a special form of bacterial collective motion, i.e., bacterial swarming in two dimensions. We will introduce some organization principles known in bacterial swarming and discuss potential means of controlling its dynamics. The simplicity and controllability of 2D bacterial behavior during swarming would allow experimental examination of theory predictions on general collective motion.
\end{abstract}

Keywords: bacterial swarming; biofilm; flagellar motility; gliding motility; biological self-organization; emergent behavior

\section{INTRODUCTION}

Collective motion is the coordinated self-organized movement of many individuals arising from physical, chemical, or social interactions between these individuals [1]. Collective motion is ubiquitous in nature. Familiar examples include fish schooling [2], bird flocking [3,4], and insect swarming [5]. At microscale, animal cells and microorganisms also display collective motion, such as cancer invasion and tissue development [6], phytoplankton (algae) blooms in surface waters [7], amoebae aggregation under starvation [8], and bacterial swarming during the formation of biofilms and fruiting bodies $[9,10]$. Collective motion is thought to confer an advantage for defending predators [2,3] and for adapting to the environment $[9,10]$.

Despite the vast differences in length scale and propulsion mechanism, collective motion across biological systems shares a similar feature: global order arises spontaneously from local interactions between individuals that do not have access to global information. Intriguingly, the traits of individuals determine the emergent global order in a non-intuitive manner; change of individuals' traits may lead to dramatically different collective behavior. Therefore, a central aim in the study of biological collective motion is to reveal its organization principles, i.e., how the traits of individuals give rise to the emergent behavior at population level. This question is not only important to biology, but also of great interest to other disciplines, such as physics, engineering, and computer science. For example, biological collective motion provides insights for understanding the physics of self-organization in non-equilibrium systems [1]; organization principles revealed in biological collective motion have inspired the design of aerial robots that can perform autonomous group flights [11], the design of swarming robots that can self-assemble in a programmable manner $[12,13]$, and the implementation of more efficient data mining algorithms using swarm intelligence [14].

To understand how individual traits give rise to the emergent behavior in collective motion, it is often desirable to control the behavior of individuals. While the behavior of multicellular organisms is complex, microorganisms are simple and new techniques from biology, physics and engineering have become available to control their collective behavior. Here we will focus on a special form of bacterial collective motion, i.e., bacterial swarming. Bacterial swarming occurs on surfaces and is 
often two-dimensional (2D). Studies of bacterial swarming have fueled the theory development for general selfpropelled particle systems, biological self-organization, and active fluids [1,15-18]. In particular, the simplicity and controllability of $2 \mathrm{D}$ bacterial behavior during swarming would allow timely examination of theory predictions by experiments. In the following sessions we will give an overview of bacterial collective motion, introduce bacterial swarming, and discuss potential means of controlling collective dynamics in bacterial swarms.

\section{OVERVIEW OF BACTERIAL COLLECTIVE MOTION}

The best known example of bacterial collective motion is chemotactic waves of bacteria migrating in bulk fluids across soft agar plates [19], which result from the chemotactic response of cells to the global chemical gradients dynamically generated by the migrating cells. Chemotactic waves have been studied in quantitative details in microfluidic channels or microcapillaries; for example, see Ref. [20]. Similar to chemotactic waves, bacteria can form traveling bands as a result of aerotaxis towards favorable oxygen concentrations in a selfgenerated oxygen gradient [21]. Bacterial collective motion at high densities has been studied in quasi-2D environments, such as in free-standing liquid films [2224], in sessile liquid drops [25,26], in confined droplets [27], and in microfluidic channels [28]. Bacterial collective motion in $2 \mathrm{D}$ has been primarily studied in bacterial swarms (to be discussed in details below). Recently, it was reported that certain bacterial species can self-assemble into rotating dynamic clusters at air-liquid or liquid-solid interface due to hydrodynamic interactions $[29,30]$.

\section{BACTERIAL COLLECTIVE MOTION IN TWO DIMENSIONS}

When grown on moist surfaces, many bacterial species are able to form a densely packed colony in which millions or more cells move across the surface in a collective manner. The colony is mostly mono-layered, so the motion of cells is two-dimensional or quasi-twodimensional. The colony is enclosed by a thin layer of liquid film or slimy matrix produced by cells themselves, which provide the milieu to support cell motility. This specialized form of 2D surface translocation exhibited by motile bacteria is called swarming. Bacterial swarms are one of the simplest biological systems displaying collective motion. Bacterial collective motion as manifested by swarming is of ecological and medical significance, because it is relevant to biofilm development [10,31], fruiting body formation [9], microbial dispersal and coexistence [32], as well as pathogenesis and tissue invasion [33].

\section{Swarming of flagellated bacteria}

Swarming was first observed with Proteus by Hauser [34], and it has long been studied in numerous bacterial species propelled by flagellar motility [34-36]. Flagella are long, thin, helical filaments appended on cell surface and they act as helical propellers to drive cell's motion through fluids [37,38]. Within hours contacting a moist nutrientrich surface, cells elongate, make more flagella, secrete wetting agents, and swarm across the surface in coordinated packs within a thin layer of fluid [39-41]. Swarming and biofilm formation are inversely regulated by pathways that enable cells to choose between swimming or entering sessile state [10]. Cells close to the swarm center gradually become sessile and produce extracellar matrix [42,43], leading to biofilm formation. For reviews on the genetics, biochemistry and physiology of flagellated swarming, see Refs. [10,35,36,44-47].

The motion pattern of swarming is similar across bacterial species. Swarm cells are densely packed but they do not jam; instead, the cells exhibit smooth collective motion in the form of clusters, swirls, and jets with a length scale of tens to hundreds of microns [48-50] (Figure 1A and D). Individual cells move at an average speed comparable to the swimming speed in bulk fluid [49]. This fact is surprising, given that cells experience frequent collisions by neighbors due to the high cell density. With an elongated shape, cells tend to move bidirectionally along the long axis of the cell body, and sideways maneuver are rare [52]. Interestingly, cells occasionally reverse moving direction upon the switching of flagellar rotation. Escherichia coli swarm cells reverses direction every $1.5 \mathrm{~s}$ [52]. The reversal period follows exponential distribution with a mean comparable to the mean run time of swimming [53], suggesting that E. coli may have adapted its chemotaxis system to control flagella motor switching during directional reversals. Alternatively the reversal may be a result of passive flipping of flagella due to cell-cell collisions [54] Directional reversal has also been observed in Paenibacillus dendritiformis swarming, in which case the reversal period distribution is unimodal but not exponential [55] (Figure 1B and E). Directional reversal may be a consequence of the elongated cell shape and crowded environment in bacterial swarms, since a rod-shaped cell would be easier to move backwards than to choose a new direction randomly when it decides to change direction in a dense crowd [17]. Chemotaxis is not required for swarming: cells of Salmonella lacking the chemotaxis response regulator, CheY, swarm perfectly well, provided that mutations in the motor protein FliM enable transi- 

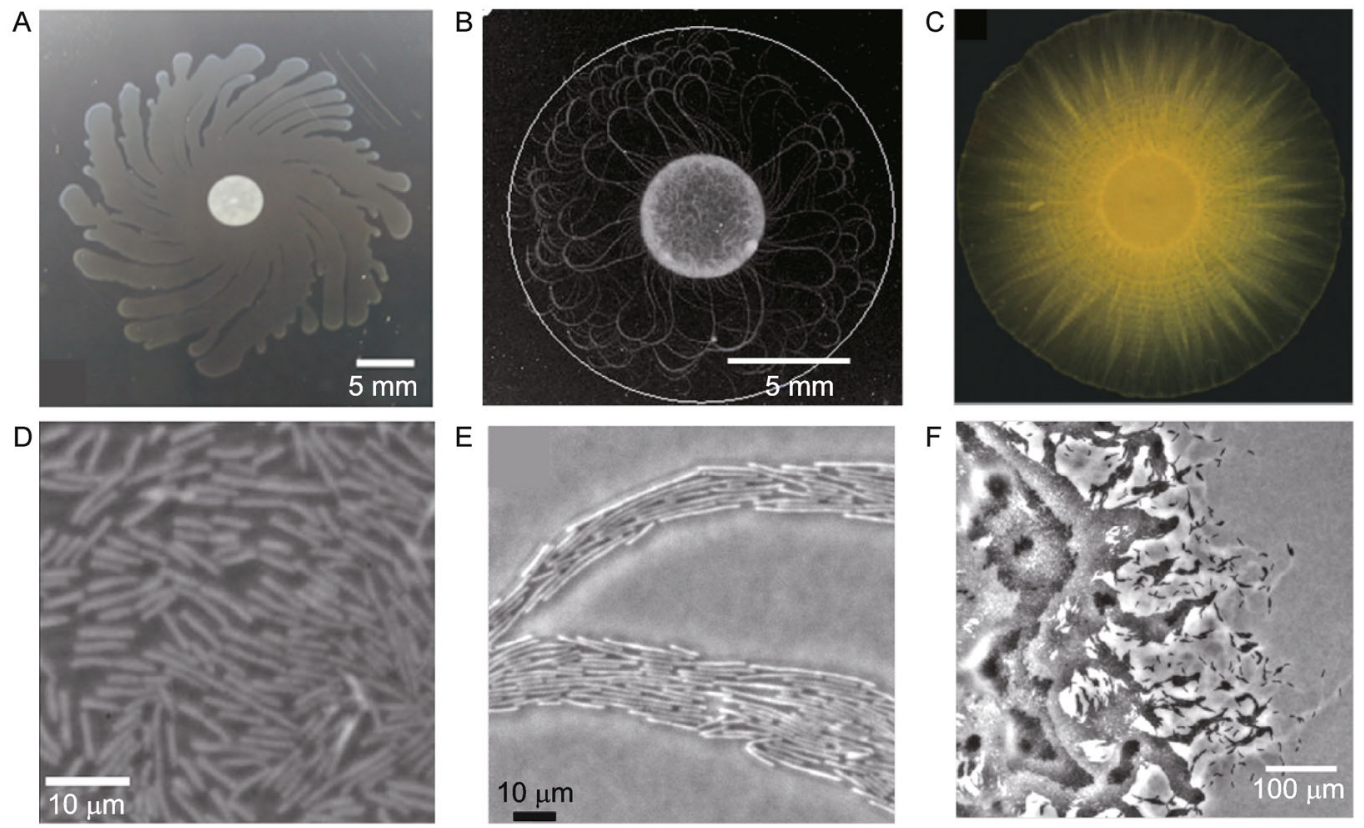

Figure 1. Swarming in diverse bacterial species. (A) Image of an E. coli swarm. Colony branches curve in a clockwise direction as a result of unidirectional fluid flows at colony edge. Adapted from Wu et al. [51] with permission. (B) Image of Paenibacillus dendritiformis swarming colony. Scale is approximate. Adapted from Be'er et al. [55] with permission. (C) Image of Myxococcus xanthus swarming colony. Adapted from [76] with permission. (D) Zoomed-in view of the advancing edge of an E. coli swarm. Multicellular clusters are evident. Adapted from a video hosted on the website of Berg lab (http://www.rowland.harvard.edu/labs/ bacteria/movies/index.php) with permission. (E) Zoomed-in view of an advancing branch of a Paenibacillus dendritiformis colony. Adapted from Be'er et al. [55] with permission. (F) Zoomed-in view of the advancing edge of a Myxococcus xanthus colony. Scale is approximate. Multicellular rafts, clusters and multilayered mounds are evident. Adapted from [77] with permission.

tions between clockwise $(\mathrm{CW})$ and counter-clockwise (CCW) states [56]. It has been reported that swarm cells exhibit giant density fluctuations [50], display scaleinvariant correlation of velocity and orientation within dynamic clusters [57], and the cells move in a manner similar to Lévy walk [58]. These behaviors are related to dynamic clustering in swarms and may be conserved among swarms of different bacterial species.

The collective dynamics in swarming is primarily governed by steric interaction between cells $[59,60]$. Hydrodynamic interaction may be a key factor as well $[49,61]$, because swarm cells are in fact swimming within a thin liquid film whose thickness is a few microns [62]. Water is drawn by cells from the substrate most likely via osmotic flows $[63,64]$; this water forms the swarm fluid film that covers the entire colony. Cells interact strongly with the swarm fluid film: the fluidic environment supports flagellar motility, while flagellar rotation strongly agitates the swarm fluid and generates rapid flows with speeds up to tens of micron per second $[51,63]$. Fluid flows driven by flagellar motility can facilitate material transport, promote social interactions, and affect the collective motion in swarms [51] (Chen C., Liu S., Wu Y., unpublished data).

\section{Swarming of non-flagellated bacteria}

Some non-flagellated bacteria were found to exhibit collective surface translocation in many ways similar to the swarming of flagellated bacteria. These bacteria are propelled by various motility mechanisms, including retraction of type IV pili (such as Pseudomonas and Myxobacteria) [65-67], slime secretion (such as Cyanobacteria) [68], and gliding with hidden engines (such as Myxobacteria and Bacteriodetes that include Cytophaga, Flavobacteria, and Bacteriodes) [9,69]. Gliding of Myxobacteria involves slime secretion and helical motion of motor proteins along cell surface [70,71]. Gliding mechanisms of Bacteriodetes are less well understood [69]; for Flavobacteria, its gliding machinery involves helical flow of surface proteins and has been identified as a rotary motor driven by proton motive force [72-74]. In microbiology literature, collective surface translocation of species propelled by type IV pili and hidden engines are 
often referred to as twitching and gliding, respectively [36]. However, the term "swarming" has been widely adopted to describe collective surface translocation of bacteria (flagellated or non-flagellated) in studies that model these phenomena and that focus on collective motion in a general sense, because the details of motility machinery can often be neglected in these studies [1].

Despite the difference in motility mechanisms, the motion pattern of gliding bacteria during swarming is similar to that of flagellated bacteria. Cells form a densely packed $2 \mathrm{D}$ or quasi-2D colony and display large scale coherent motion, such as rafts, clusters, and multilayered mounds [75] (Figure 1C and F). These elongated and flexible cells tend to reverse moving direction in a regular manner [69,77]. This regular reversal is required for Myxobacteria to swarm; it facilitates orientational ordering and ensures smooth cellular flow within a dense crowd whose density is near jamming threshold [77,78]. A similar function may be conferred by directional reversal in swarming of flagellated bacteria [17]. Notably, Myxobacteria were found to regulate reversal period using an internal biochemical clock when predating prey bacteria or during starvation [79], and the changes of reversal behavior lead to the formation of rippling waves [80], streams [81,82], as well as 3D fruiting bodies [83]. Short-ranged contact-mediated interactions are the primary interactions between gliding bacteria, as suggested by a number of cell-based biomechanical models that have successfully explained some key aspects of collective motion of gliding bacteria [59,77,81,83-88].

\section{CONTROLLING BACTERIAL COLLECTIVE MOTION IN TWO DIMENSIONS: A PERSPECTIVE}

Individual cell's behavioral pattern gives rise to emergent collective behavior often in an unexpected manner. For example, variation of directional reversal frequencies in Myxobacteria can change collective behavior from swarming to rippling [80] or to streaming [82]. On the other hand, models of self-propelled particle systems and active fluids suggest that behavioral parameters of individuals are often critical to collective dynamics $[11,15,16,18]$. For example, Chate et al. studied the role of noise in active nematic systems and found that correlation length diverges (i.e., quasi-long-range order emerges) below a critical noise strength [89]; McCandlish et al. and Hinz et al. modeled mixtures of self-propelled particles with different motilities, and found that such systems displayed phase separation [90] and could give rise to dynamical phases [91]; Nagai et al. found that selfpropelled particles with memory of past trajectories (i.e., with underdamped angular dynamics) displayed a rich variety of phases not observed in systems without memory, such as vortex lattices and active foam [92] To understand the connections between individual cell's behavior and the emergent collective behavior, and to verify model predictions in experimental systems of bacterial collective motion, it is desirable to control the behavior of individual cells as well as their physicochemical environment.

A growing number of techniques have become available to control the behavior of bacteria. New development in synthetic biology has allowed the building of genetic circuits that can modify the way cells behave and interact during collective motion. For example, incorporation of a quorum sensing circuit to the chemotactic pathway of $E$. coli generated programmable stripe formation [93]. It is also possible to control the behavior of cells with light, using optogenetics tools [94] and photosensitizers. For example, using a photosensitizer, $\mathrm{Lu}$ et al. investigated the response of collective motion to increased noise level [95]. Bacillus subtilis swarms were supplemented with the photosensitizer protoporphyrin IX. Upon light illumination, the photodynamic action of protoporphyrin IX generated reactive oxygen species, which disrupted the function of flagellar motors and caused cells to become more tumbly, thus increasing the noise level. The authors measured the collective speed of cells during light illumination and demonstrated loss of collective motion due to increased noise level [95].

The physicochemical environment of swarm cells can be controlled using microfluidic devices. Swiecicki et al. used a microfluidic device to control the height of the liquid environment of $E$. coli swarm cells and recovered the key features of collective swarming dynamics, such as cluster formation [60]. Recently Liu et al. used a macroscopically sized microfluidic device to observe the 3D colony expansion of $B$. subtilis under controlled chemical environment [96]. This technique may be used to study bacterial collective motion under controlled chemical environment.

With these and more new techniques becoming available, the time is ripe for a deeper understanding of bacterial collective motion and for the examination of collective motion models in a wider parameter space.

\section{ACKNOWLEDGEMENTS}

This work is supported by funding from CUHK Direct Research Grants (Nos. 4053019, 4053079 and 4053130) the Research Grants Council of HKSAR (RGC Ref. No. CUHK 409713), and from the National Natural Science Foundation of China (No. NSFC 21473152).

\section{COMPLIANCE WITH ETHICS GUIDELINES}

The author Yilin Wu declare that he has no conflict of interests. 
This article does not contain any studies with human or animal subjects performed by the author.

\section{REFERENCES}

1. Vicsek, T. and Zafeiris, A. (2012) Collective motion. Phys. Rep., 517, $71-140$

2. Ioannou, C. C., Guttal, V. and Couzin, I. D. (2012) Predatory fish select for coordinated collective motion in virtual prey. Science, 337, 12121215

3. Cavagna, A., Cimarelli, A., Giardina, I., Parisi, G., Santagati, R., Stefanini, F. and Viale, M. (2010) Scale-free correlations in starling flocks. Proc. Natl. Acad. Sci. USA, 107, 11865-11870

4. Attanasi, A., Cavagna, A., Del Castello, L., Giardina, I., Jelic, A., Melillo, S., Parisi, L., Pohl, O., Shen, E. and Viale, M. (2015) Emergence of collective changes in travel direction of starling flocks from individual birds' fluctuations. J. R. Soc. Interface, 12, 20150319

5. Bazazi, S., Buhl, J., Hale, J. J., Anstey, M. L., Sword, G. A., Simpson, S. J. and Couzin, I. D. (2008) Collective motion and cannibalism in locust migratory bands. Curr. Biol., 18, 735-739

6. Friedl, P. and Gilmour, D. (2009) Collective cell migration in morphogenesis, regeneration and cancer. Nat. Rev. Mol. Cell Biol., $10,445-457$

7. Hallegraeff, G. M. (1993) A review of harmful algal blooms and their apparent global increase. Phycologia, 32, 79-99.

8. Tyson, J. J. and Murray, J. D. (1989) Cyclic AMP waves during aggregation of Dictyostelium amoebae. Development, 106, 421-426

9. Kaiser, D. (2007) Bacterial swarming: a re-examination of cellmovement patterns. Curr. Biol., 17, R561-R570

10. Verstraeten, N., Braeken, K., Debkumari, B., Fauvart, M., Fransaer, J., Vermant, J. and Michiels, J. (2008) Living on a surface: swarming and biofilm formation. Trends Microbiol., 16, 496-506

11. Vasarhelyi, G., Viragh, C., Somorjai, G., Tarcai, N., Szörenyi, T., Nepusz, T., Vicsek, T. (2014) Outdoor flocking and formation flight with autonomous aerial robots. In IROS, 2014 IEEE/RSJ Inter. Confer. 3866-3873

12. Gross, R., Bonani, M., Mondada, F. and Dorigo, M. (2006) Autonomous self-assembly in swarm-bots. Robotics. IEEE Transactions on, 22, 1115-1130

13. Rubenstein, M., Cornejo, A. and Nagpal, R. (2014) Programmable selfassembly in a thousand-robot swarm. Science, 345, 795-799

14. Martens, D., Baesens, B. and Fawcett, T. (2011) Editorial survey: swarm intelligence for data mining. Mach. Learn., 82, 1-42

15. Ramaswamy, S. (2010) The mechanics and statistics of active matter. Annu. Rev. Condens. Matter Phys., 1, 323-345

16. Koch, D. L. and Subramanian, G. (2011) Collective hydrodynamics of swimming microorganisms: living fluids. Annu. Rev. Fluid Mech., 43, 637-659

17. Wu, Y., Jiang,Y., Kaiser, A. D. and Alber, M. (2011) Self-organization in bacterial swarming: lessons from myxobacteria. Phys. Biol., 8, 055003

18. Marchetti, M. C., Joanny, J. F., Ramaswamy,S., Liverpool,T. B., Prost, J., Rao, M. and Simha, R. A. (2013) Hydrodynamics of soft active matter. Rev. Mod. Phys., 85, 1143-1189

19. Berg, H. C. (2004) E. coli in Motion. New York: Springer-Verlag

20. Saragosti, J., Calvez, V., Bournaveas, N., Perthame, B., Buguin, A. and Silberzan, P. (2011) Directional persistence of chemotactic bacteria in a traveling concentration wave. Proc. Natl. Acad. Sci. USA, 108, 16235-
16240

21. Mazzag, B. C., Zhulin, I. B. and Mogilner, A. (2003) Model of bacterial band formation in aerotaxis. Biophys. J., 85, 3558-3574

22. Wu, X.-L. and Libchaber, A. (2000) Particle diffusion in a quasi-twodimensional bacterial bath. Phys. Rev. Lett., 84, 3017-3020

23. Sokolov, A., Aranson, I. S., Kessler, J. O. and Goldstein, R. E. (2007) Concentration dependence of the collective dynamics of swimming bacteria. Phys. Rev. Lett., 98, 158102

24. Sokolov, A. and Aranson, I. S. (2012) Physical properties of collective motion in suspensions of bacteria. Phys. Rev. Lett., 109, 248109

25. Dombrowski, C., Cisneros, L., Chatkaew, S., Goldstein, R. E. and Kessler, J. O. (2004) Self-concentration and large-scale coherence in bacterial dynamics. Phys. Rev. Lett., 93, 098103

26. Cisneros, L. H., Kessler, J. O., Ganguly, S. and Goldstein, R. E. (2011) Dynamics of swimming bacteria: transition to directional order at high concentration. Phys. Rev. E Stat. Nonlin. Soft Matter Phys., 83, 061907

27. Lushi, E., Wioland, H. and Goldstein, R. E. (2014) Fluid flows created by swimming bacteria drive self-organization in confined suspensions. Proc. Natl. Acad. Sci. USA, 111, 9733-9738

28. Wensink, H. H., Dunkel, J., Heidenreich, S., Drescher, K., Goldstein, R. E., Löwen, H. and Yeomans, J. M. (2012) Meso-scale turbulence in living fluids. Proc. Natl. Acad. Sci. USA, 109, 14308-14313

29. Petroff, A. P., Wu, X.-L. and Libchaber, A. (2015) Fast-moving bacteria self-organize into active two-dimensional crystals of rotating cells. Phys. Rev. Lett., 114, 158102

30. Xiao, C., Xiang, Y., Mingcheng, Y. and Zhang, H. P. (2015) Dynamic clustering in suspension of motile bacteria. EPL, 111, 54002

31. López, D., Vlamakis, H. and Kolter, R. (2010) Biofilms. Cold Spring Harb. Perspect. Biol., 2, a000398

32. Kerr, B., Riley, M. A., Feldman, M. W. and Bohannan, B. J. M. (2002) Local dispersal promotes biodiversity in a real-life game of rock-paperscissors. Nature, 418, 171-174

33. Struthers, J. K. and Westran, R. P. (2003) Clinical Bacteriology. 192. Florida: CRC Press

34. Hauser, G. (1885) Über Fäulnisbakterien und deren Beziehung zur Septicämie. Leipzig: F. G. W. Vogel

35. Harshey, R. M. (2003) Bacterial motility on a surface: many ways to a common goal. Annu. Rev. Microbiol., 57, 249-273

36. Kearns, D. B. (2010) A field guide to bacterial swarming motility. Nat. Rev. Microbiol., 8, 634-644

37. Berg, H. C. (2003) The rotary motor of bacterial flagella. Annu. Rev. Biochem., 72, 19-54

38. Lauga, E. and Powers, T. R. (2009) The hydrodynamics of swimming microorganisms. Rep. Prog. Phys., 72, 096601

39. Allison, C., Lai, H.-C., Gygi, D. and Hughes, C. (1993) Cell differentiation of Proteus mirabilis is initiated by glutamine, a specific chemoattractant for swarming cells. Mol. Microbiol., 8, 53-60

40. Harshey, R. M. and Matsuyama, T. (1994) Dimorphic transition in Escherichia coli and Salmonella typhimurium: surface-induced differentiation into hyperflagellate swarmer cells. Proc. Natl. Acad. Sci. USA, 91, 8631-8635

41. Partridge, J. D. and Harshey, R. M. (2013) More than motility: Salmonella flagella contribute to overriding friction and facilitating colony hydration during swarming. J. Bacteriol., 195, 919-929

42. Hamze, K., Autret, S., Hinc, K., Laalami, S., Julkowska, D., Briandet, R., Renault, M., Absalon, C., Holland, I. B., Putzer, H., et al. (2011) Single-cell analysis in situ in a Bacillus subtilis swarming community identifies distinct spatially separated subpopulations differentially 
expressing hag (flagellin), including specialized swarmers. Microbiology, 157, 2456-2469

43. Tremblay, J. and Déziel, E. (2010) Gene expression in Pseudomonas aeruginosa swarming motility. BMC Genomics, 11, 587

44. Henrichsen, J. (1972) Bacterial surface translocation: a survey and a classification. Bacteriol Rev, 36, 478-503

45. Allison, C. and Hughes, C. (1991) Bacterial swarming: an example of prokaryotic differentiation and multicellular behaviour. Sci. Prog., 75, 403-422

46. McCarter, L. L. (2004) Dual flagellar systems enable motility under different circumstances. J. Mol. Microbiol. Biotechnol., 7, 18-29

47. Copeland, M. F. and Weibel, D. B. (2009) Bacterial swarming: a model system for studying dynamic self-assembly. Soft Matter, 5, 1174 1187

48. Zhang, H. P., Be'er, A., Smith, R. S., Florin, E.-L. and Swinney, H. L. (2009) Swarming dynamics in bacterial colonies. EPL, 87, 48011

49. Darnton, N. C., Turner, L., Rojevsky, S. and Berg, H. C. (2010) Dynamics of bacterial swarming. Biophys. J., 98, 2082-2090

50. Zhang, H. P., Be'er, A., Florin, E.-L. and Swinney, H. L. (2010) Collective motion and density fluctuations in bacterial colonies. Proc. Natl. Acad. Sci. USA, 107, 13626-13630

51. Wu, Y., Hosu, B. G. and Berg, H. C. (2011) Microbubbles reveal chiral fluid flows in bacterial swarms. Proc. Natl. Acad. Sci. USA, 108, 41474151

52. Turner, L., Zhang, R., Darnton, N. C. and Berg, H. C. (2010) Visualization of flagella during bacterial swarming. J. Bacteriol., 192, 3259-3267

53. Berg, H. C. and Brown, D. A. (1972) Chemotaxis in Escherichia coli analysed by three-dimensional tracking. Nature, 239, 500-504

54. Cisneros, L., Dombrowski, C., Goldstein, R. E., Kessler, J. O. (2006) Reversal of bacterial locomotion at an obstacle. Phys. Rev. E Stat. Nonlin. Soft Matter Phys. 73:03090173: 030901

55. Be'er, A., Strain, S. K., Hernández, R. A., Ben-Jacob, E. and Florin, E.L. (2013) Periodic reversals in Paenibacillus dendritiformis swarming. J. Bacteriol., 195, 2709-2717

56. Mariconda, S., Wang, Q. and Harshey, R. M. (2006) A mechanical role for the chemotaxis system in swarming motility. Mol. Microbiol., 60, $1590-1602$

57. Chen, X., Dong, X., Be'er, A., Swinney, H. L. and Zhang, H. P. (2012) Scale-invariant correlations in dynamic bacterial clusters. Phys. Rev. Lett., 108, 148101

58. Ariel, G., Rabani, A., Benisty, S., Partridge, J. D., Harshey, R. M. and Be'er, A. (2015) Swarming bacteria migrate by Lévy Walk. Nat. Commun., 6, 8396

59. Peruani, F., Deutsch, A. and Bär, M. (2006) Nonequilibrium clustering of self-propelled rods. Phys. Rev. E Stat. Nonlin. Soft Matter Phys., 74, 030904

60. Swiecicki, J.-M., Sliusarenko, O. and Weibel, D. B. (2013) From swimming to swarming: Escherichia coli cell motility in twodimensions. Integr. Biol. (Camb), 5, 1490-1494

61. Drescher, K., Dunkel, J., Cisneros, L. H., Ganguly, S. and Goldstein, R. E. (2011) Fluid dynamics and noise in bacterial cell-cell and cellsurface scattering. Proc. Natl. Acad. Sci. USA, 108, 10940-10945

62. Zhang, R., Turner, L. and Berg, H. C. (2010) The upper surface of an Escherichia coli swarm is stationary. Proc. Natl. Acad. Sci. USA, 107, 288-290

63. Wu, Y. and Berg, H. C. (2012) Water reservoir maintained by cell growth fuels the spreading of a bacterial swarm. Proc. Natl. Acad. Sci.
USA, $109,4128-4133$

64. Ping, L., Wu, Y., Hosu, B. G., Tang, J. X. and Berg, H. C. (2014) Osmotic pressure in a bacterial swarm. Biophys. J., 107, 871-878

65. Semmler, A. B. T., Whitchurch, C. B. and Mattick, J. S. (1999) A reexamination of twitching motility in Pseudomonas aeruginosa. Microbiology, 145, 2863-2873

66. Nudleman, E. and Kaiser, D. (2004) Pulling together with type IV pili. J. Mol. Microbiol. Biotechnol., 7, 52-62

67. Anyan, M. E., Amiri, A., Harvey, C. W., Tierra, G., Morales-Soto, N., Driscoll, C. M., Alber, M. S. and Shrout, J. D. (2014) Type IV pili interactions promote intercellular association and moderate swarming of Pseudomonas aeruginosa. Proc. Natl. Acad. Sci. USA, 111, 1801318018

68. Hoiczyk, E. and Baumeister, W. (1998) The junctional pore complex, a prokaryotic secretion organelle, is the molecular motor underlying gliding motility in cyanobacteria. Curr. Biol., 8, 1161-1168

69. McBride, M. J. (2004) Cytophaga-flavobacterium gliding motility. J. Mol. Microbiol. Biotechnol., 7, 63-71

70. Kaiser, D. (2003) Coupling cell movement to multicellular development in myxobacteria. Nat. Rev. Microbiol., 1, 45-54

71. Nan, B., McBride, M. J., Chen, J., Zusman, D. R. and Oster, G. (2014) Bacteria that glide with helical tracks. Curr. Biol., 24, R169-R173

72. Nelson, S. S., Bollampalli, S. and McBride, M. J. (2008) SprB is a cell surface component of the Flavobacterium johnsoniae gliding motility machinery. J. Bacteriol., 190, 2851-2857

73. Nakane, D., Sato, K., Wada, H., McBride, M. J. and Nakayama, K. (2013) Helical flow of surface protein required for bacterial gliding motility. Proc. Natl. Acad. Sci. USA, 110, 11145-11150

74. Shrivastava, A., Lele, P. P. and Berg, H. C. (2015) A rotary motor drives Flavobacterium gliding. Curr. Biol., 25, 338-341

75. Kaiser, D. and Warrick, H. (2014) Transmission of a signal that synchronizes cell movements in swarms of Myxococcus xanthus. Proc. Natl. Acad. Sci. USA, 111, 13105-13110

76. Jelsbak, L. and Kaiser, D. (2005) Regulating pilin expression reveals a threshold for S motility in Myxococcus xanthus. J. Bacteriol., 187, 2105-2112

77. Wu, Y., Kaiser, A. D., Jiang, Y. and Alber, M. S. (2009) Periodic reversal of direction allows Myxobacteria to swarm. Proc. Natl. Acad. Sci. USA, 106, 1222-1227

78. Kudrolli, A. (2010) Concentration dependent diffusion of self-propelled rods. Phys. Rev. Lett., 104, 088001

79. Igoshin, O. A., Goldbeter, A., Kaiser, D. and Oster, G. (2004) A biochemical oscillator explains several aspects of Myxococcus xanthus behavior during development. Proc. Natl. Acad. Sci. USA, 101, 15760 15765

80. Berleman, J. E., Scott, J., Chumley, T. and Kirby, J. R. (2008) Predataxis behavior in Myxococcus xanthus. Proc. Natl. Acad. Sci. USA, 105, 17127-17132

81. Alber, M. S., Kiskowski, M. A. and Jiang,Y. (2004) Two-stage aggregate formation via streams in myxobacteria. Phys. Rev. Lett., 93, 068102

82. Thutupalli, S., Sun, M., Bunyak, F., Palaniappan, K. and Shaevitz, J. W. (2015) Directional reversals enable Myxococcus xanthus cells to produce collective one-dimensional streams during fruiting-body formation. J. R. Soc. Interface, 12, 20150049

83. Sozinova, O., Jiang, Y., Kaiser, D. and Alber, M. (2005) A threedimensional model of myxobacterial aggregation by contact-mediated interactions. Proc. Natl. Acad. Sci. USA, 102, 11308-11312 
84. Wu, Y., Jiang, Y., Kaiser, D. and Alber, M. (2007) Social interactions in myxobacterial swarming. PLoS Comput. Biol., 3, e253

85. Janulevicius, A., van Loosdrecht, M. C. M., Simone, A. and Picioreanu, C. (2010) Cell flexibility affects the alignment of model myxobacteria. Biophys. J., 99, 3129-3138

86. Peruani, F., Starruß, J., Jakovljevic, V., Søgaard-Andersen, L., Deutsch, A. and Bär, M. (2012) Collective motion and nonequilibrium cluster formation in colonies of gliding bacteria. Phys. Rev. Lett., 108, 098102

87. Janulevicius, A., van Loosdrecht, M. and Picioreanu, C. (2015) Shortrange guiding can result in the formation of circular aggregates in myxobacteria populations. PLoS Comput. Biol., 11, e1004213

88. Balagam, R. and Igoshin, O. A. (2015) Mechanism for collective cell alignment in Myxococcus xanthus bacteria. PLoS Comput. Biol., 11, e1004474

89. Chaté, H., Ginelli, F. and Montagne, R. (2006) Simple model for active nematics: quasi-long-range order and giant fluctuations. Phys. Rev. Lett., 96, 180602

90. McCandlish, S. R., Baskaran, A. and Hagan, M. F. (2012) Spontaneous segregation of self-propelled particles with different motilities. Soft
Matter, 8, 2527-2534.

91. Hinz, D. F., Panchenko, A., Kim, T.-Y. and Fried, E. (2014) Motility versus fluctuations in mixtures of self-motile and passive agents. Soft Matter, 10, 9082-9089

92. Nagai, K. H., Sumino, Y., Montagne,R., Aranson, I. S. and Chaté, H. (2015) Collective motion of self-propelled particles with memory. Phys. Rev. Lett., 114, 168001

93. Liu, C., Fu, X., Liu, L., Ren, X., Chau, C. K. L., Li, S., Xiang, L., Zeng, H., Chen, G., Tang, L. H., et al. (2011) Sequential establishment of stripe patterns in an expanding cell population. Science, 334, 238241

94. Deisseroth, K. (2011) Optogenetics. Nat. Methods, 8, 26-29

95. Lu, S., Bi, W., Liu, F., Wu, X., Xing, B. and Yeow, E. K. (2013) Loss of collective motion in swarming bacteria undergoing stress. Phys. Rev. Lett., 111, 208101

96. Liu, J., Prindle, A., Humphries, J., Gabalda-Sagarra, M., Asally, M., Lee, D. Y., Ly, S., Garcia-Ojalvo, J. and Süel, G. M. (2015) Metabolic co-dependence gives rise to collective oscillations within biofilms. Nature, 523, 550-554 